\title{
Procedimiento para la evaluación del desempeño ambiental: aplicación em el sector energético cubano
}

\section{Environmental performance evaluation procedure: application in the cuban energy sector}

\author{
Frank Medel-González ${ }^{1}$ \\ Lourdes García-Ávila ${ }^{1}$ \\ Cecilia Hernández ${ }^{2}$ \\ Mailyn Medel-González ${ }^{3}$
}

\begin{abstract}
Resumen: El artículo propone un abordaje estructurado para evaluar el desempeño ambiental a través de un procedimiento que establece una línea de acción para realizar la evaluación del desempeño ambiental de empresas cubanas, integrando indicadores ambientales que responden a las principales políticas y estrategias. La propuesta tiene como objetivo presentar contribuciones teóricas para la evaluación del desempeño ambiental empresarial en el entorno de Cuba, para complementar la evaluación con medidas de resultados del desempeño ambiental y garantizar la eficacia de las políticas, estrategias y metas. La propuesta surge de la carencia de instrumentos metodológicos que permitan la selección, agrupamiento, integración y evaluación de indicadores ambientales que permitan evaluar el desempeño ambiental garantizando la eficacia de las políticas, estrategias y metas. El procedimiento facilita la selección de indicadores ambientales tomando como base las estrategias y principales cuestiones ambientales, modelos de integración y herramientas tales como ISO 14031, el Balanced Scorecard (BSC) y métodos para la toma de decisiones multicriterio, específicamente el Analytic Network Process. También se propone en el trabajo la obtención de un valor único de desempeño ambiental global, con base en el nivel de cumplimiento de las metas y los indicadores ambientales definidos inicialmente. Para la validación del procedimiento se aplicó en cuatro pequeñas y medianas centrales eléctricas, donde además de evaluar el desempeño ambiental, fue posible identificar los puntos críticos y oportunidades de mejora para re direccionar los esfuerzos y resolver los principales puntos críticos.
\end{abstract}

Palabras claves: Evaluación del desempeño ambiental; Indicadores ambientales; Balanced Scorecard; Analytic Network Process.

\begin{abstract}
The present article proposes a structured approach to assessing environmental performance through a procedure that establishes a course of action to complete the evaluation of corporate environmental performance, integrating environmental indicators that respond to major policies and strategies. The proposal aims to present theoretical contributions to the assessment of corporate environmental performance in the Cuban environment, to complement the assessment of environmental performance outcome measures to ensure the effectiveness of policies, strategies and targets. The procedure facilitates the selection of environmental indicators based on strategies and major environmental issues, integrating standards and tools such as the ISO 14000, the Balanced Scorecard (BSC), and multi-criteria methods. The study also proposes an overall environmental performance based on the level of compliance with the goals of environmental indicators defined for displaying a single value in the evaluation of environmental performance. For validation, the procedure was applied in four small-and medium-sized power stations, where environmental indicators were defined, calculated, and weighted using the Analytic Network Process (ANP) multi-criteria method, which was later integrated to the global index of environmental performance to be evaluated according the numerical value. Also, the application facilitated the identification of critical points and improvement opportunities to redirect efforts to address the main weaknesses.
\end{abstract}

Keywords: Environmental performance evaluation; Environmental indicators; Balanced Scorecard; Analytic Network Process.

\footnotetext{
${ }^{1}$ Departamento de Ingeniería Industrial, Universidad Central Marta Abreu de Las Villas - UCLV, CEP 54830, Santa Clara, Cuba, e-mail: frankmedel@uclv.edu.cu; lourdes@uclv.edu.cu

${ }^{2}$ Departamento Engenharia de Produção, Universidade Federal Fluminense - UFF, CEP 24220-240, Niterói, Rio de Janeiro, RJ, Brazil, e-mail: ctoledo@id.uff.br

${ }^{3}$ Empresa Eléctrica de Villa Clara, CEP 52290, Santa Clara, Villa Clara, Cuba, e-mail: mailyn@elecvcl.une.cu

Recibido Mayo 25, 2012 - Aceptado Jul. 28, 2015

Apoyo financiero: Parte de esta investigación esta soportada por el proyecto "Desarrollo de herramientas informáticas para soportar la gestión del desarrollo sustentable y el Cambio Climático en territorios y ecosistemas cubanos" (código P211LH001013), perteneciente al programa nacional, Cambio Climático en Cuba: impactos, mitigación y adaptación de la Agencia de Medio Ambiente.
} 


\section{Introducción}

Desde el año 1987 con la definición de Desarrollo Sostenible se ha apreciado en el escenario internacional, en todos los sectores de la economía, una concientización por la protección del medio ambiente. Este fenómeno ha sido impulsado principalmente por la legislación asociada, derivada de la necesidad de conservación de recursos naturales y reducción de impactos asociados al desempeño ambiental de las organizaciones.

Muchas empresas están buscando modos de entender, demostrar y mejorar su desempeño ambiental, esto se puede alcanzar con una gestión eficaz de aquellos elementos que puedan tener un impacto significativo en el medio ambiente, en el desarrollo de sus actividades, productos y servicios.

En este escenario, el tratamiento de la información ambiental y la obtención de un número limitado de indicadores claves se convierten en elementos críticos para el éxito en una organización, ayudando a los directivos en el proceso de toma de decisiones ambientales.

Según Henri \& Journeault (2008) la importancia de la medición se refiere a la atención dedicada por las empresas para cuantificar diversas cuestiones ambientales. Investigaciones previas han identificado cuatro dimensiones de desempeño ambiental que los indicadores ambientales deben medir: interna, externa, procesos y el resultado (Lober, 1996; Ilinitch et al., 1998).

Esta necesidad creciente de mejora continua del desempeño ambiental de las organizaciones y el incremento gradual de la legislación regulatoria asociada a la protección del medio ambiente, ha estimulado la investigación en este campo como, Luz et al. (2006), Ramos \& Melo (2006), Gunningham (2009), Sellitto et al. (2010) y Raupp (2012).

En los últimos treinta años el pensamiento y la acción ambiental cubana ha crecido y se ha perfeccionado, en el aspecto legislativo, político, educativo, científico, tecnológico, social y económico; influyendo positivamente en el proceso de toma de decisiones (García-Fernández, 2002). Sin embargo, aún existen deficiencias en relación con la evaluación del desempeño ambiental, como proceso de gestión interna de las organizaciones cubanas, que ayude a seleccionar, recopilar, integrar y evaluar indicadores ambientales. Los mejores intentos se recogen en las investigaciones de Parra (2005), Broche-Fernández \& Ramos-Gómez (2010), Isaac-Godínez et al. (2010) y Sagastume-Gutiérrez et al. (2012); pero aún no se evidencia un modelo o procedimiento que contabilice el desempeño organizacional basado en indicadores operacionales y de gestión, que brinden información condensada del desempeño respecto a las metas organizacionales, facilitando la identificación de puntos críticos y oportunidades de mejoras.
El objetivo general del artículo, es presentar contribuciones teóricas para la evaluación del desempeño ambiental empresarial en el entorno cubano, a través de un procedimiento que oriente en la selección e integración de indicadores ambientales y la evaluación haciendo uso del índice global de desempeño ambiental (IGDA); que facilite la identificación de puntos críticos y oportunidades de mejora.

El tema energía ha sido una prioridad del gobierno cubano desde el mismo triunfo de la Revolución. Para la aplicación del procedimiento se escogió el sector energético específicamente las centrales eléctricas de la generación distribuida (GD).

Las centrales eléctricas que se estudiaron se encuentran en las categorías de pequeñas y medianas centrales eléctricas (PyMCE) de acuerdo al volumen de generación que producen.

Luego de la introducción, el artículo presenta: (i) revisión teórica sobre la evaluación del desempeño e indicadores ambientales, índices agregados y métodos multi-criterio; (ii) metodología de la pesquisa; (iii) resultados de la aplicación del procedimiento propuesto; (iv) conclusiones y trabajos futuros.

\section{Evaluación del desempeño ambiental e indicadores ambientales}

El desempeño ambiental es considerado un concepto derivado de la gestión ambiental. Según NC-ISO 14031 (ISO, 2005) son los resultados medibles de la gestión que hace una organización de sus actividades, productos y/o servicios que puede interactuar con el medio ambiente. Las organizaciones no solo deben conocer su desempeño ambiental sino evaluarlo para identificar avances y retrocesos en la relación de la organización con el medio ambiente. La evaluación del desempeño ambiental (EDA) está basada en la máxima, "lo que no puede ser medido, no puede ser gestionado". Según NC-ISO 14031 (ISO, 2005) la EDA es el proceso utilizado para facilitar las decisiones de la dirección con respecto al desempeño ambiental de la organización mediante la selección de indicadores, la recopilación y el análisis de datos, la evaluación de la información comparada con los criterios de desempeño ambiental, los informes $\mathrm{y}$ comunicaciones, las revisiones periódicas y las mejoras de este proceso. Isaac-Godínez et al. (2010) plantea que la evaluación del desempeño debe estar relacionada con las características de la organización y de sus procesos sustantivos, ser capaz de estructurar y proporcionar la información para la toma de decisiones, y la comunicación de una gestión eficaz en función del cuidado del medio ambiente. La evaluación del desempeño ambiental debe ser multidimensional y no limitarse a informes, pues estos no integran los 
múltiples factores que afectan el desempeño ambiental empresarial (Pearson \& Barnes, 1999).

Los indicadores de desempeño ambiental son la base de la EDA y miden el desempeño ambiental actual o pasado de una organizacion y lo compara contra las metas definidas por la direccion (Ormazabal \& Larrañaga, 1999; Jasch, 2000; Herva et al., 2011), representan la cuantificación de la efectividad y eficiencia de las acciones ambientales con un conjunto de métricas (Neely et al., 1995) y hacen referencia a la medida de interacción entre la organización y el medio ambiente (Olsthoorn et al., 2001). También representan las mediciones cualitativas y cuantitativas, financieras o no financieras, que proporcionan información importante sobre el impacto ambiental, cumplimiento regulatorio, las relaciones con las stakeholders y los sistemas organizacionales (Henri \& Journeault, 2008)

Las estructuras analíticas de los sistemas de indicadores ambientales más usadas, son las siguientes:

1. Indicadores según modelo de Presión-EstadoRespuesta (PER), introducido por la Organización para la Cooperación Económica y Desarrollo 1994 (OECD, siglas en inglés).

2. Indicadores de desempeño ambiental y de condición ambiental NC-ISO 14031 (ISO, 2005).

3. Indicadores para comunicar el desempeño ambiental según Global Reporting Initiative (GRI, 2006).

Algunos de los indicadores ambientales empresariales más usados son recogidos en Ormazabal \& Larrañaga (1999), NC-ISO 14031 (ISO, 2005), GRI (2006), Rao et al. (2009) y Herva et al. (2011).

\subsection{Sistemas de Medición del desempeño ambiental}

Un sistema de medición del desempeño (SMD) puede ser definido como un conjunto de métricas usadas para cuantificar la eficiencia y efectividad de las acciones (Neely et al., 1995). Según Tangen (2005) un exitoso SDM es grupo de medidas de desempeño que proveen a la organización de información útil, que ayudan a gestionar, controlar, planificar y desarrollar las actividades emprendidas por la organización.

Tangen (2005) define tres clases de SMD: tercera clase, donde son usadas medidas de desempeño tradicional y los requerimientos son bastante bajos; segunda clase, tiene una vista más balanceada del desempeño incluyendo medidas no financieras y considerando diferentes horizontes de tiempo y niveles dentro de la organización; primera clase, son los más avanzados cubriendo gran cantidad de requerimientos, capaz de explicar relaciones causales en la organización, considerando las necesidades de los stakeholders e integrando bases de datos y sistemas de información.

En la bibliografía se evidencian investigaciones que hace uso de SMD ambiental en organizaciones. Campos \& Selig (2002) proponen una metodología Sistema de Gestión y Evaluación del Desempeño Ambiental (SGADA) que permite la inclusión de cuestiones ambientales en la estrategia de la organización. Su principal objetivo es identificar evolución o estancamiento en relación al desempeño ambiental de los procesos. SGADA hace uso del Balanced Scorecard para tratar las cuestiones estratégicas relacionadas a la gestión ambiental y agregan una perspectiva a la estructura tradicional del BSC, la perspectiva ambiental. Existen varias tendencias a la hora de incluir los temas ambientales y sociales en el $B S C$, la primera es integrar los aspectos ambientales y sociales dentro de las cuatro perspectivas tradicionales del BSC (Epstein \& Wisner, 2001; Figge et al., 2002; Möller \& Schaltegger, 2005); otra variante es agregar perspectivas a la estructura tradicional (Bieker \& Waxenberger, 2001; Hubbard, 2009; Medel et al., 2011), otra es construir un BSC ambiental independiente y la cuarta variante es hacer una combinación de las tres anteriores. Sin embargo el uso de la estructura tradicional del $B S C$ es mejor aceptada por las organizaciones y permite la inclusión a lo largo de todas sus perspectivas de los diferentes temas ambientales y puede ser implementado como un proceso evolutivo, véase (Möller \& Schaltegger, 2005).

Ramos \& Melo (2006) proponen un SDM y hacen uso de evaluaciones por cuestionarios en el sector militar portugués y determinan un índice agregado de desempeño ambiental que refleja la relación entre las prácticas de gestión ambiental y las organizaciones militares, ayudando a los decisores a comprender el perfil medioambiental militar.

Luz et al. (2006) y mas reciente Sellitto et al. (2010), proponen y aplican un método para medir el desempeño ambiental cuyo principal objetivo es capturar, con indicadores integrados, la complejidad presente en sistemas ambientales y como esta se manifiesta de forma sistémica. Para esto dividen el impacto ambiental de la operación, en cinco subsistemas, atribuyéndoles importancias relativas a través del $A H P$, en el impacto global y describiendo este proceso mediante indicadores que son evaluados por expertos a través de la escala de Likert. Posteriormente agregan los indicadores y forman un índice global que varía entre $0 \%$ y $100 \%$. La principal limitación de este modelo es que se apoya solo del juicio de los especialistas en oposición a las mediciones que se valen de medidas físicas de variables de campo o de modelos matemáticos, que se usan como base para el cálculo de los indicadores (Sellitto et al., 2010). Esta propuesta está dirigida principalmente a evaluar el 
desempeño ambiental basado en el impacto ambiental producido por la empresa.

La investigación de Broche-Fernández \& Ramos-Gómez (2010) se basa en un diagnóstico del desempeño ambiental organizacional que incluye la determinación de un indicador de evaluación integral que toma en cuenta un total de diez variables que son evaluadas de forma cualitativa haciendo uso de una escala numérica equivalente (de 1 a 10), lo que es reconocido por los autores como una limitante en esta propuesta.

Raupp (2012) define un modelo de autoevaluación a partir de un referente teórico. Hace uso del Método de Evaluación de Indicadores de Sostenibilidad (MAIS, siglas en portugués) propuesto por Oliveira (2002) basado en la aplicación de cuestionarios que revela la realización o no de acciones para alcanzar las cuestiones ambientales relevantes.

La generalidad de las investigaciones consultadas en la literatura tienen en común que hacen uso de información cualitativa: derivada de la aplicación de encuestas sobre las prácticas ambientales en organizaciones (Ramos \& Melo, 2006; Raupp, 2012); otras a partir de la valoración de indicadores y variables, haciendo énfasis en los impactos ambientales provocados, según el juicio de los expertos, (Luz et al., 2006; Sellitto et al., 2010; Broche-Fernández \& Ramos-Gómez, 2010). La propuesta de Campos \& Selig (2002) incluye elementos importantes como: objetivos, metas, indicadores de desempeño e identificación de requisitos legales, todo esto combinado con un BSC de cinco perspectivas. Según Möller \& Schaltegger (2005) usando las cuatro perspectivas del BSC convencional aumenta la aceptacion de las mejoras. Tambien el tema de las partes interesadas es poco abordado en esta última investigación, aunque no deja de ser la mas completa de las analizadas.

\section{2 Índices agregados}

Existe gran tendencia a la construcción de índices agregados en el área ambiental y de la sostenibilidad. Las stakeholders prefieren los índices y además le permite a la empresa no dar información detallada del funcionamiento en sí del sistema, pero si dan una idea de su desempeño (Zhou et al., 2006). Ejemplos de la literatura consultada, por solo citar algunos, lo demuestran Puolamaa et al. (1996), Chiang \& Lai (2002), Damjan \& Glavic (2005), Ramos \& Melo (2006), Singh et al. (2007), Blanc et al. (2008), Hubbard (2009), Sellitto et al. (2010) y Broche-Fernández \& Ramos-Gómez (2010).

Para la construcción de índices agregados de cualquier índole es necesario una serie de pasos entre los que se encuentran: la selección de los indicadores, la homogenización, la normalización, la ponderación y la agregación.
- Selección: proceso de decisión de los indicadores que integrarán el índice agregado.

- Homogenización: es el paso de llevar los indicadores seleccionados de naturaleza diferente a un mismo criterio ya sea de maximizar o minimizar.

- Normalización: los indicadores que componen los índices están distribuidos sobre diferentes categorías por lo que es necesaria una unidad común o equivalente. Algunos de los métodos más usados en la literatura para la normalización son: Z-score, normalización lineal, Min.-Máx. y otros como la normalización difusa.

- Ponderación: proceso para determinar y asignar las importancias relativas de los indicadores basado en criterio de expertos. Según Paoli \& Moraes (2011), los métodos multi-criterio están siendo utilizados en evaluaciones de impactos ambientales. Una de las técnicas más usadas para ponderar índices ha sido AHP (Analytic Hierarchy Process).

- Agregación: es el resumen de la información en un valor único, logrando el índice deseado. Según Diewert (1976) varias reglas y formas funcionales para la agregación de índices compuestos se han desarrollado en la literatura. Algunos de los métodos más usados según Zhou et al. (2006) son: el método de la utilidad aditiva, producto ponderado, media geométrica ponderada.

\subsection{Métodos multi-criterio}

AHP es una técnica de decisión multi-criterio, la cual fue desarrollada por Saaty (1980). Según Hernández et al. (2010) el AHP es el método de decisión multi-criterio más referenciado en la literatura en los últimos 20 años. Otros como Hermans et al. (2008) plantean que esta ha sido una de las técnicas más usadas para la evaluación de los pesos de los indicadores ambientales tomando como ejemplos: Indoor Environment Index (Chiang \& Lai, 2002) y Environmental Friendliness (Puolamaa et al., 1996). También Saaty (2003) plantea que los pesos de los indicadores de sostenibilidad son generalmente obtenidos haciendo uso del método de decisión $A H P$.

En los últimos años varias investigaciones han ilustrado la preferencia de asignación sobre ciertos atributos por encima o por debajo de otros siempre que la información no esté completa. El campo de la ingeniería ambiental y la sostenibilidad no han escapado a esta preferencia, algunos ejemplos de la 
aplicación del $A H P$ en estas áreas se pueden apreciar en Damjan \& Glavic (2005), Luz et al. (2006), Singh et al. (2007) y Hernández et al. (2010).

A pesar de la amplia aceptación del $A H P$ en la construcción de índices, este brinda una visión poco realista de fenómenos naturales ya que parte de la independencia que debe de existir entre los niveles y alternativas del modelo. En ocasiones estas relaciones suelen ser más complejas, con un mayor número de relaciones e interrelaciones convirtiendo el modelo jerárquico en una estructura compleja.

Más reciente, en 1996, fue desarrollado por el propio Saaty, el método ANP (Analytic Network Process). $A N P$ provee una herramienta para lidiar con las decisiones sin asumir la independencia de los elementos de un nivel superior a los elementos de un nivel inferior y sobre la independencia de los elementos dentro de un nivel en una jerarquía. El $A N P$ hace una extensión del método $A H P$ para los problemas con dependencias y retroalimentación entre los criterios usando el enfoque de la "súper-matriz" (Saaty, 1996). Según Hernández (2010), ANP no obedece al axioma de independencia entre criterios o influencia entre alternativas.

Con respecto a la estructura de la decisión, Saaty $\&$ Saaty (2003) observa que el $A N P$ hace uso de las redes sin la necesidad de especificar niveles. Como en el $A H P$, la dominación o la importancia relativa de influencia es un concepto central, la ampliamente divulgada teoría multi-criterio $A H P$ es un caso especial del $A N P$.

El ANP está compuesto por dos partes: 1) control de la jerarquía o la red de objetivos y criterios que controlan las interacciones del sistema bajo estudio y 2) muchas subredes de influencias entre todos los elementos y grupos del problema, uno por cada criterio de control.

Una red puede ser generada a partir de una jerarquía incrementando gradualmente las interconexiones, logrando las conexiones de los grupos como se deseen. Algunos grupos tienen elementos que se relacionan entre ellos lo que hace que presenten dependencia interna.

En los últimos años un aumento de la aplicación del $A N P$ se evidencia en la producción científica; algunos ejemplos se pueden apreciar en Wang et al. (2006), Yüksel \& Dagdeviren (2010), Hernández et al. (2010), Hsu et al. (2011) y Lee et al. (2011), todos estos en diferentes áreas del conocimiento lo que da idea del éxito que ha tenido esta herramienta a nivel mundial.

\section{Metodología de pesquisa}

Según Lakatos \& Marconi (1986), el problema de pesquisa se relaciona al análisis de un tema o laguna del conocimiento que todavía no tiene solución. En este caso el problema científico, la evaluación del desempeño ambiental, ha sido ampliamente abordado en la literatura internacional (véase: Dias-Sardinha \& Reijnders, 2005; Luz et al., 2006; Ramos \& Melo, 2006; Gunningham, 2009; Sellitto et al., 2010; Raupp, 2012; Comoglio \& Botta, 2012) pero en el ámbito cubano se evidencian carencias en la EDA apoyado por la escaza publicación de articulos cientiicos, teóricos y de aplicación práctica.

La propuesta presentada es un procedimiento para la EDA (Figura 1), permite conducir la selección, establecimiento e integración de indicadores, que estén acorde con las necesidades que tiene la dirección de las empresas, de que la evaluación del desempeño sea complementada con medidas internas, externas (stakeholders), de los procesos y los resultados, para saber si las políticas, estrategias y metas ambientales son efectivas.

Basados en el carácter multidimensional del desempeño ambiental, se hace uso de la estructura del Balanced Scorecard para el diseño de un índice global que incluya las áreas relevantes de la organización y los stakeholders, combinado con métodos multi-criterio como el $A N P$ y $A H P$.

El método de pesquisa fue el mixto (Creswell, 2007) que combina un levantamiento cualitativo y cuantitativo de datos ambientales.

Para realizar la pesquisa primeramente fue utilizado un levantamiento cualitativo. La colecta de los datos precisó de un estudio teórico sobre los procesos de evaluación de desempeño ambiental y las mejores prácticas.

Para el estudio práctico fueron seleccionadas cuatro PyMCE, dos de fuel-oil (CEF1, CEF2) y dos de diésel (CED1, CED2) de la red de generación distribuida de la provincia de Villa Clara, Cuba. Para esta selección piloto se tuvo en cuenta la disponibilidad de informaciones para el cálculo de posibles indicadores y representatividad de los dos tipos de centrales existentes.

La obtención de los datos primarios fue mediante entrevistas a directivos para clarificar las estrategias ambientales, trabajo en grupo para identificar los aspectos e impactos ambientales en cada central hasta definir y agrupar los indicadores ambientales utilizando las perspectivas del $B S C$.

Posteriormente se pasó a la segunda fase de la pesquisa dónde el procedimiento de evaluación comenzó a ser implementado. En esta fase fue de gran importancia el levantamiento cuantitativo que permitió colectar y establecer las relaciones entre los indicadores propuestos para la aplicación del $A N P y$ $A H P$, lo que posibilitó la ponderación de los indicadores y el cálculo del índice global de desempeño.

Finalmente fue realizada la comparación de los valores calculados de los índices para la interpretación y validación de los resultados por el equipo de trabajo, permitiendo cuantificar el desempeño ambiental e identificar puntos críticos en el desempeño ambiental de las PyMCE. 


\section{Fase 1: Análisis estratégico ambiental}

\subsection{Organización del estudio}

\subsection{Clarificar estrategias ambientales}

Fase 5: Revisión y mejora

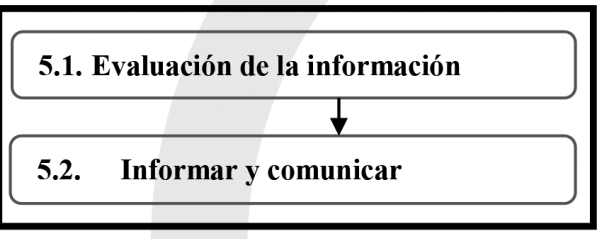

Fase 4: Cálculo del IGDA

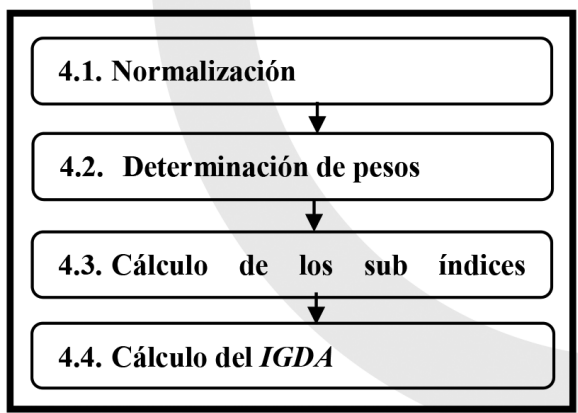

Fase 2: Inventario de los procesos

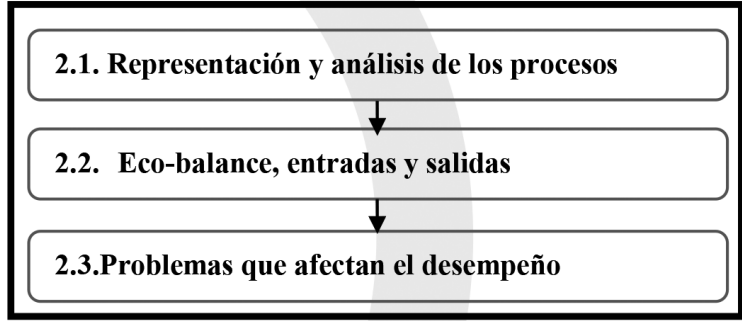

Fase 3: Selección de Indicadores ambientales

\begin{tabular}{|l|}
\hline 3.1. Selección inicial de los indicadores \\
\hline 3.2. Clasificación y agrupación de los indicadores \\
\hline 3.3. Confección de planillas de los indicadores \\
\hline
\end{tabular}

Figura 1. Procedimiento propuesto para la evaluación del desempeño ambiental. Fuente: Elaboración propia.

\section{Resultados}

Para ejemplificar el procedimiento propuesto en la Figura 1, se realizó la aplicación para evaluar el desempeño ambiental en cuatro PyMCE en el contexto empresarial cubano. A continuación se muestran cómo se desarrollaron las cinco fases con los principales resultados.

\subsection{Fase I: Análisis estratégico ambiental}

En esta primera fase se definió el alcance de la investigación y se llevó a cabo la preparación del grupo de trabajo. Una de las metas a alcanzar fue el compromiso de la dirección con la aplicación del procedimiento. También se seleccionaron los expertos que intervinieron en la recopilación de los datos e informaciones, selección de indicadores y en la emisión de los juicios para ponderar los indicadores. El grupo de expertos quedo conformado por siete especialistas: cuatro en gestión ambiental, dos profesores investigadores y el especialista principal de calidad de las CE; en total cuatro Doctores y tres Masters, los cuales integraron los paneles para la aplicación del ANP y AHP.

En esta fase se emplearon técnicas de análisis y recopilación de información, indicadores técnicos-organizativos y organigramas. Se clarificaron las estrategias, aspectos, políticas, objetivos e impactos ambientales más significativos de las CE.

Otro elemento importante fue la identificación de las stakeholders y los requisitos regulatorios asociados a las PyMCE. Además se realizó el análisis del entorno en que se desenvuelve la empresa, con sus amenazas y oportunidades como factores externos, y sus fortalezas y debilidades como factores internos.

\subsection{Fase 2. Inventario de los procesos}

En esta fase se profundizó en el proceso de generación y se realizó la representación gráfica del mismo (Figura 2), identificando las principales entradas de recursos, energía, agua, combustible, aceites y las salidas: generación de energía como producto, residuos líquidos y emisiones a lo largo de todo el proceso.

Del mismo modo quedaron identificados los principales problemas que presentan las cuatro $\mathrm{CE}$, siendo estos: las emisiones no contabilizadas de gases a la atmósfera, gran cantidad y variedad de residuales líquidos como aguas oleosas, aguas residuales, lodos generados del proceso de purificación del fuel-oil y aceites usados; los altos niveles de ruido en áreas de las $\mathrm{CE}$, producido por los motores generadores, y las deficiencias detectadas en el cumplimiento de los 
requisitos regulatorios, por stakeholders reguladoras que han realizado auditorías.

De igual forma se detallaron los principales aspectos e impactos ambientales (ver principales Tabla 1) de cada central. En todos los casos se evaluaron los impactos según la metodología de Conesa-Fernández (1995) ampliamente aceptada, facilitando la clarificación de las importancias de los impactos. Esta fase facilita la familiarización del equipo de trabajo y los expertos con el proceso de generación.

\subsection{Fase 3. Selección de los indicadores ambientales}

Tomando como base las fases anteriores, las estrategias, las políticas, aspectos e impactos ambientales, los expertos luego de reuniones seleccionaron inicialmente

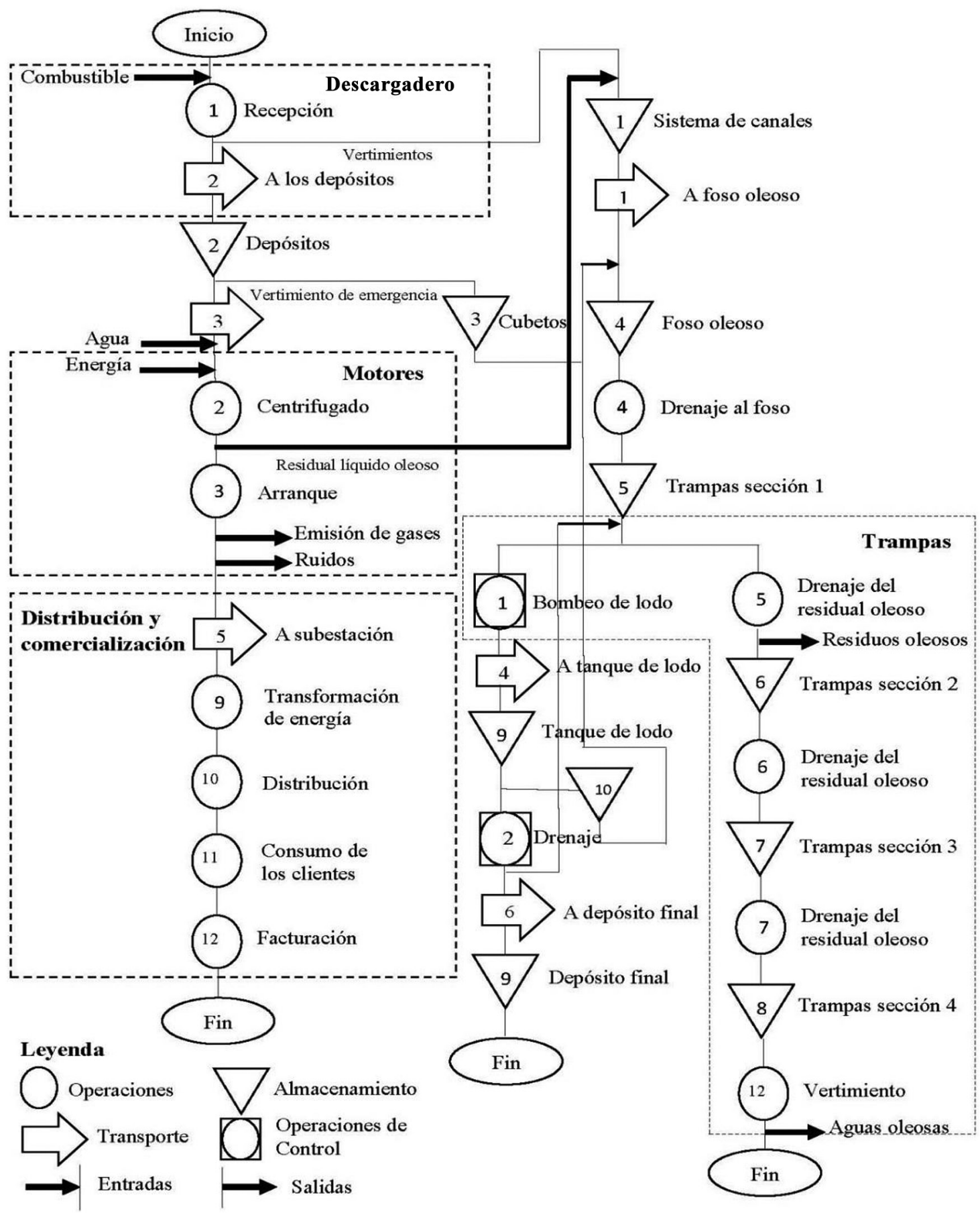

Figura 2. Representación del proceso de generación e identificación de entradas y salidas. Fuente: Elaboración propia. 
Tabla 1. Aspectos e impactos ambientales identificados en las actividades de las centrales.

\begin{tabular}{|c|c|c|}
\hline Actividad & Aspecto asociado & Impacto ambiental \\
\hline \multirow{6}{*}{ Proceso de generación } & Consumo de agua & $\begin{array}{l}\text { Reducción de la disponibilidad del } \\
\text { recurso agua para otros usos }\end{array}$ \\
\hline & Generación de altos niveles de ruido & $\begin{array}{l}\text { Afectación a la salud de los } \\
\text { trabajadores / Contaminación acústica } \\
\text { de la zona }\end{array}$ \\
\hline & Vertimiento de residuales líquidos & $\begin{array}{l}\text { Contaminación de las aguas } \\
\text { superficiales y subterráneas }\end{array}$ \\
\hline & Consumo de portadores energéticos & $\begin{array}{l}\text { Reducción de la disponibilidad para } \\
\text { otros usos }\end{array}$ \\
\hline & Emanaciones de gases a la atmósfera & Contaminación del medio ambiente \\
\hline & Generación de desechos peligrosos & $\begin{array}{l}\text { Contaminación del suelo a las aguas } \\
\text { superficiales }\end{array}$ \\
\hline Purificación de fuel oil & $\begin{array}{l}\text { Generación de lodos en el proceso de } \\
\text { centrifugado }\end{array}$ & Contaminación por mal manejo \\
\hline $\begin{array}{l}\text { Reparación y mantenimiento de } \\
\text { equipos }\end{array}$ & Generación de desechos peligrosos & Contaminación por mal manejo \\
\hline
\end{tabular}

Fuente: Elaboración propia.

un total de 27 indicadores ambientales. Para esto se apoyaron en bibliografía como: Ormazabal \& Larrañaga (1999), NC-ISO 14031 (ISO, 2005), Rao et al. (2009) y Herva et al. (2011) en visitas técnicas a las diferentes centrales eléctricas.

Los indicadores se agruparon en tres clasificaciones derivadas de:

- El desempeño ambiental operacional de la entidad derivable de la eficiencia de las actividades y el consumo de recursos de la empresa.

- La efectividad de las medidas organizativas o sea del nivel de gestión de la entidad, para corregir el desempeño ambiental. Está relacionado con el control de los impactos ambientales, cumplimiento de metas, capacitación de personal.

- El estado de legalidad ambiental de las actividades empresariales, en términos de la obtención de licencias, cumplimiento de leyes ambientales generales.

Estos indicadores se agruparon en las cuatro perspectivas del BSC (Finanzas, Stakeholders, Procesos internos, Crecimiento y Aprendizaje). Vale destacar que se sustituyó la tradicional perspectiva de Clientes por la de Stakeholders haciéndola más abarcadora, varias investigaciones han adoptado la perspectiva de Stakeholders véase Dias-Sardinha \& Reijnders (2005), Hubbard (2009) y Hsu et al. (2011).

Posteriormente se ordenaron según el criterio de los expertos, para valorar cuáles según su importancia debían incluirse; limitando el número de indicadores seleccionados a cinco como máximo por perspectiva. En la Tabla 2 se muestran los indicadores ambientales que mejor responden a los principales aspectos e impactos de las CE según la valoración del grupo de expertos.

En esta fase deben quedar definidos el método de cálculo, unidad de medida, frecuencia de medición, objetivo estratégico al que responde, metas y las relaciones causales con otros indicadores. Las metas de cada indicador deben ser razonables, desafiantes y alcanzables en un período estratégico. Para fijar las metas de desempeño de los indicadores, los expertos junto con el grupo de trabajo utilizaron tres formas para su determinación:

1. Comportamiento histórico del indicador (en caso de existir previamente) o análisis histórico de las variables que conforman el indicador.

2. Análisis de indicadores ambientales de otras $\mathrm{CE}$ con reconocimiento ambiental (tipo de Benchmarking).

3. Derivados de los requisitos regulatorios asociados a las actividades de generación.

\subsection{Fase 4. Cálculo del índice global de desempeño ambiental}

En esta fase se procede a calcular el IGDA (Figura 3) para el que es necesario: normalizar los indicadores, determinar los pesos de los indicadores y perspectivas, calcular los subíndices, para las cuatro perspectivas del $B S C$ y luego integrarlos brindando una visión integral del desempeño ambiental empresarial a través del IGDA.

Para el cálculo del índice global de desempeño ambiental se propuso la Fórmula 1, la que tiene como variables el peso de cada perspectiva, el peso 
del indicador dentro de la perspectiva y el valor normalizado del indicador.

$$
I G D A=\sum_{j=1}^{j=\sum_{i=1}=n} W p_{j} W i_{i j} R_{i j}
$$

IGDA: Índice global de desempeño ambiental. Valor acotado entre 0 y 1 .

$W p_{j}$ : El peso de la perspectiva $\mathrm{j}$.

$W_{i j}$ : El peso del indicador i en la perspectiva $\mathrm{j}$.
$R_{i j}$ : Valor normalizado del indicador i de la perspectiva j.

Para el cálculo de los subíndices de cada perspectiva se utilizó la Fórmula 2, que permite ilustrar cómo se están comportando los indicadores de determinada perspectiva.

$$
I P_{j}=\sum_{i=1}^{i=n} W i_{i j} R_{i j}
$$

$I P_{j}:$ Índice de la perspectiva $\mathrm{j}$.

$W i_{i j}$ : El peso relativo del indicador i en la perspectiva $\mathrm{j}$.

\begin{tabular}{|c|c|c|}
\hline Perspectivas & Código & Indicadores \\
\hline \multirow{4}{*}{ Finanzas } & F1 & $\begin{array}{l}\text { Costo de generación del MW ( } \$ / \mathrm{MW}) \text { : es el resultado de la multiplicación de la cantidad } \\
\text { de combustible consumido (ton) por el precio ( } \$ \text { ton), dividido por la cantidad de MW } \\
\text { generados (Operacional) }\end{array}$ \\
\hline & F2 & $\begin{array}{l}\text { Inversiones ambientales ( } \$ \text { año): mide el grado de ejecución monetaria de las inversiones } \\
\text { de carácter ambiental o que tengan un impacto positivo sobre el medio ambiente en el } \\
\text { período analizado (Gestión) }\end{array}$ \\
\hline & F3 & $\begin{array}{l}\text { Costos ambientales por caracterización (\$/año): son el resultado de la contratación de } \\
\text { servicios ambientales a terceros (Gestión) }\end{array}$ \\
\hline & F4 & $\begin{array}{l}\text { Multas impuestas: evaluar en función de la cantidad de multas y los importes el estado } \\
\text { de la central. } 0 \text { multas } \rightarrow \text { Bien (7), } 1 \text { multa } \leq \$ 5000 \rightarrow \text { Regular (5) y } 2 \text { multas o } 1 \text { multa }> \\
\$ 5000 \rightarrow \text { Mal (3) (Legalidad) }\end{array}$ \\
\hline \multirow{5}{*}{ Stakeholders } & S1 & $\begin{array}{l}\text { Número de incidentes ambientales y/o quejas de la comunidad: Para su evaluación se } \\
\text { utiliza la siguiente escala. } 0 \rightarrow \operatorname{Bien~(7),~} 1 \rightarrow \text { Regular (5) y } 2 \text { o más } \rightarrow \text { Mal (3) (Legalidad) }\end{array}$ \\
\hline & S2 & $\begin{array}{l}\text { Deficiencias promedio detectadas en auditorías: el total de incidencias detectadas por } \\
\text { auditorías tanto internas como externas sobre el total de auditorías. } 0 \rightarrow \text { Bien (7), } 1 \text { ó } 2 \\
\rightarrow \text { Regular (5) y } 3 \text { ó más } \rightarrow \text { Mal (3) (Gestión) }\end{array}$ \\
\hline & S3 & $\begin{array}{l}\text { Cumplimiento de las regulaciones: listar los requisitos regulatorios a cumplir (leyes y } \\
\text { decretos). Asignar valor } 1 \text { a los que se cumplen totalmente, } 0,5 \text { a los que se cumplen } \\
\text { parcialmente y el valor } 0 \text { a los que no se cumplen (Legalidad) }\end{array}$ \\
\hline & S4 & $\begin{array}{l}\text { Número de auditorías internas: total de auditorías internas programadas, realizadas en el } \\
\text { periodo (Gestión) }\end{array}$ \\
\hline & S5 & $\begin{array}{l}\text { Satisfacción de stakeholders: se calcula en función de las evaluaciones realizada por las } \\
\text { stakeholders del desempeño de la unidad (Legalidad) }\end{array}$ \\
\hline \multirow{5}{*}{$\begin{array}{l}\text { Procesos } \\
\text { internos }\end{array}$} & PI1 & $\begin{array}{l}\text { Consumo específico fuel oil o diesel (g/kWh): Combustible consumido (gramos) sobre } \\
\text { los kWh generados (Operacional) }\end{array}$ \\
\hline & PI2 & $\begin{array}{l}\text { Lodos y aguas residuales generados por el consumo de combustible }+ \text { aceites: lodos } \\
\text { generados (litros) por las CE sobre la generación del periodo (MW) y aguas residuales } \\
\left(\mathrm{m}^{3}\right) \text { sobre la generación del período (Operacional) }\end{array}$ \\
\hline & PI3 & $\begin{array}{l}\text { Consumo de agua por } \mathrm{kW}\left(\mathrm{m}^{3} / \mathrm{MW}\right) \text { : total de agua consumida en el período }\left(\mathrm{m}^{3}\right) \text { sobre el } \\
\text { total generado (MW) (Operacional) }\end{array}$ \\
\hline & PI4 & $\begin{array}{l}\text { Nivel de ruido: compara los estudios realizados en el período con los valores establecidos } \\
\text { en la NC 19-01-04: } 80 \text { (Operacional) }\end{array}$ \\
\hline & PI5 & $\begin{array}{l}\text { Emisiones gaseosas: contabiliza las emisiones totales emitidas por el proceso de } \\
\text { generación resultado de los kWh generados por el factor de emisión del combustible } \\
\text { utilizado (Operacional) }\end{array}$ \\
\hline \multirow{4}{*}{$\begin{array}{l}\text { Crecimiento } \\
\text { y aprendizaje }\end{array}$} & CA1 & $\begin{array}{l}\text { Trabajadores que tienen requisitos ambientales en la descripción de sus puestos: razón de } \\
\text { trabajadores con requisitos ambientales sobre total de trabajadores (Gestión) }\end{array}$ \\
\hline & CA2 & $\begin{array}{l}\text { Trabajos de mejoras ambientales generados por trabajadores: } 2 \rightarrow \text { Bien (7), } 1 \rightarrow \text { Regular } \\
\text { (5) y } 0 \rightarrow \text { Mal (3) (Gestión) }\end{array}$ \\
\hline & CA3 & $\begin{array}{l}\text { Resultados de las encuestas realizadas a los empleados acerca de su conocimiento de los } \\
\text { asuntos ambientales de la organización: resultados de las evaluaciones realizadas por el } \\
\text { departamento de calidad a los trabajadores (Gestión) }\end{array}$ \\
\hline & CA4 & Promedio de horas de capacitación por trabajador: (Gestión) \\
\hline
\end{tabular}

Tabla 2. Indicadores ambientales definidos por perspectivas.

Fuente: Elaboración propia. 


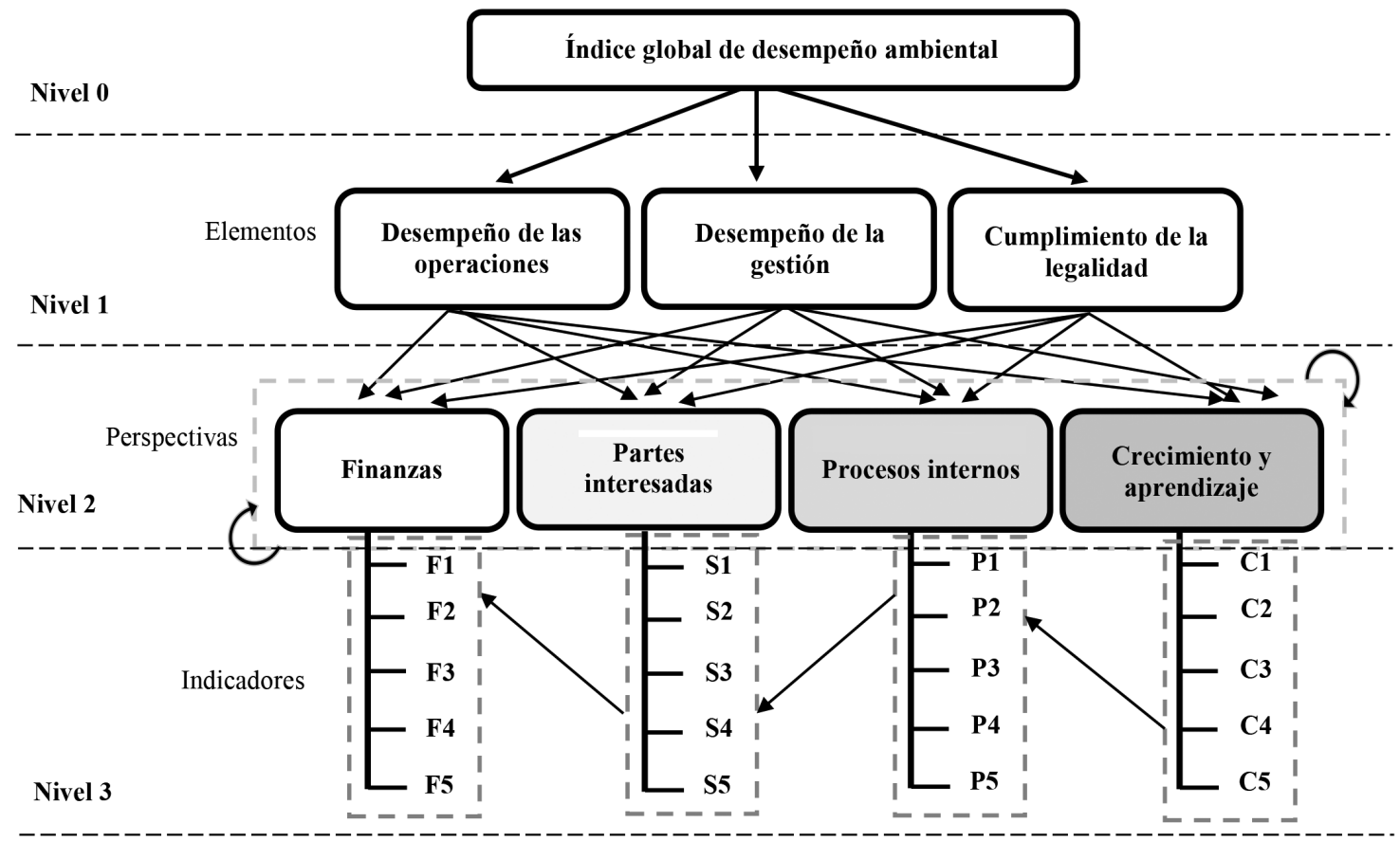

Figura 3. Estructura de la evaluación del desempeño ambiental multi-criterio basado en redes analíticas $(A N P)$. Fuente: Elaboración propia.

Tabla 3. Matriz de alcance global.

\begin{tabular}{|c|c|c|c|c|c|c|c|}
\hline & Meta & Estrategias & Perspectivas & $\mathbf{F}$ & $\mathbf{S}$ & PI & $\mathbf{C A}$ \\
\hline Meta & 0 & 0 & 0 & 0 & 0 & 0 & 0 \\
\hline Estrategias & 1 & 0 & 0 & 0 & 0 & 0 & 0 \\
\hline Perspectivas & 0 & 1 & 1 & 0 & 0 & 0 & 0 \\
\hline $\mathbf{F}$ & 0 & 0 & 1 & 0 & 1 & 1 & 1 \\
\hline $\mathbf{S}$ & 0 & 0 & 1 & 0 & 0 & 1 & 1 \\
\hline PI & 0 & 0 & 1 & 0 & 0 & 0 & 1 \\
\hline $\mathbf{C A}$ & 0 & 0 & 1 & 0 & 0 & 0 & 0 \\
\hline
\end{tabular}

Fuente: Elaboración propia.

$R_{i j}$ : Valor normalizado del indicador i de la perspectiva j.

Para el cálculo de los pesos se utilizó el software SuperDecisions desarrollado y coordinado por Saaty, que implementa el método $A N P$, disponible para descargar en el sitio (Saaty \& Saaty, 2003). Inicialmente se determinó la matriz de alcance global (Tabla 3) que identifica las relaciones existentes entre los grupos o clusters, teniendo en cuenta las relaciones existentes entre los indicadores de diferentes perspectivas del $B S C$.

Para la construcción de la matriz local (Tabla 4) se identificaron las relaciones existentes entre los nodos de los niveles cero, uno y dos.

Se identificaron las relaciones de dependencia entre los indicadores que finalmente quedaron seleccionados. Para esto se utilizó una simple matriz de correlación donde se incluyeron los 18 indicadores agrupados en las cuatro perspectivas y se les aplicó a los expertos, considerándose las relaciones, si al menos cuatro de los siete expertos coincidían. Posteriormente se construyó un mapa de dependencia de indicadores (Figura 4) que representa las relaciones identificadas, enfatizando dos tipos de relaciones, directas (en la misma perspectiva) e indirectas (con otras perspectivas).

Luego de identificar relaciones de dependencia y conformar las matrices de alcance global y local se procedió a emitir los juicios de los expertos para la aplicación del $A N P$, se realizaron las comparaciones a pares desde niveles superiores a los inferiores, para ello se emitieron los juicios de todos los especialistas para la comparación tomándose el valor modal de los juicios, en caso de empate se escogió el valor más cercano a la media de los juicios emitidos.

Primeramente se emitieron los juicios de la importancia de la gestión, legalidad y operacional para las centrales, correspondientes al nivel uno (Tabla 5). Para ello se tuvo en cuenta la inconsistencia (I) de los juicios emitidos la cual debe ser menor del $10 \%$.

De igual forma se emitieron los juicios para establecer la influencia de los elementos del nivel 


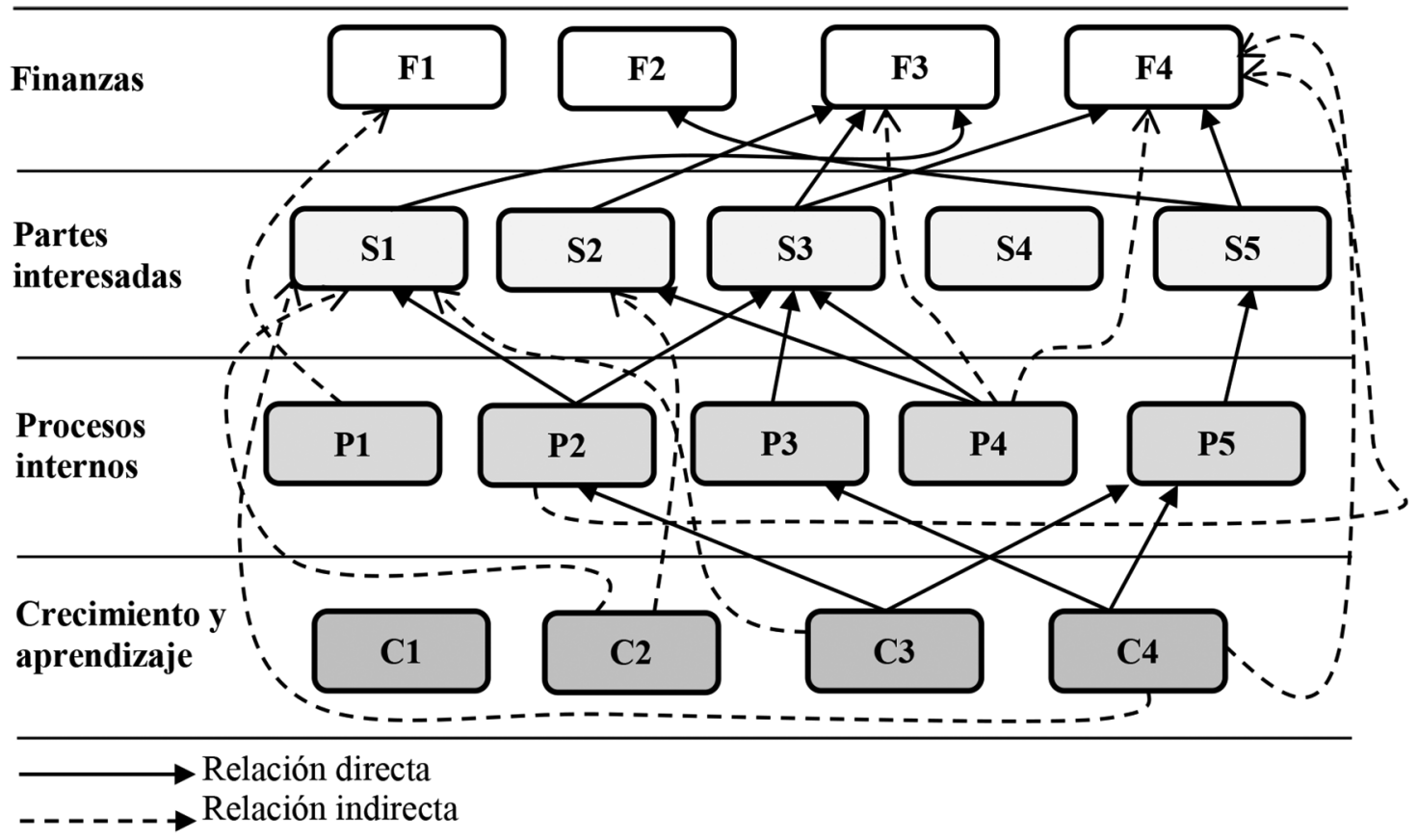

Figura 4. Relaciones de dependencia entre indicadores. Fuente: Elaboración propia.

Tabla 4. Matriz de alcance local para los grupos estrategia ambiental y perspectivas.

\begin{tabular}{cccccccccc}
\hline & & Meta & Gestión & Legalidad & Operacional & F & S & PI & CA \\
\hline Nivel 0 & Meta & 0 & 0 & 0 & 0 & 0 & 0 & 0 & 0 \\
Nivel 1 & Gestión & 1 & 0 & 0 & 0 & 0 & 0 & 0 & 0 \\
& Legalidad & 1 & 0 & 0 & 0 & 0 & 0 & 0 & 0 \\
& Operacional & 1 & 0 & 0 & 0 & 0 & 0 & 0 & 0 \\
\multirow{5}{*}{ Nivel 2} & F & 0 & 1 & 1 & 1 & 0 & 1 & 1 & 1 \\
& S & 0 & 1 & 1 & 1 & 1 & 0 & 1 & 1 \\
& PI & 0 & 1 & 1 & 1 & 1 & 1 & 0 & 1 \\
& CA & 0 & 1 & 1 & 1 & 1 & 1 & 1 & 0 \\
\hline
\end{tabular}

Fuente: Elaboración propia.

Tabla 5. Juicios emitidos entre por los expertos para los elementos.

\begin{tabular}{ccccc}
\hline I=0.0147 & Gestión & Legalidad & Operacional & Vector \\
\hline Gestión & 1 & 0,5 & 0,33 & 0,17 \\
Legalidad & 2 & 1 & 1 & 0,39 \\
Operacional & 3 & 1 & 1 & 0,44 \\
\hline
\end{tabular}

Fuente: Elaboración propia.

uno sobre las cuatro perspectivas del nivel dos del índice, conformándose un total de tres matrices.

Cada matriz contó con un total de seis juicios, las combinaciones posible para una matriz de cuatro por cuatro. La inconsistencia fue de 0,09, 0,05 y 0,08 siendo consistentes los juicios emitidos por los expertos.

Según Yüksel \& Dagdeviren (2010) las dependencias entre las perspectivas son determinadas analizando el impacto de cada perspectiva sobre todas las demás usando la comparación a pares. Lo que muestra como las perspectivas del $B S C$ no se relacionan entre sí de
Tabla 6. Pesos de las perspectivas del $B S C$.

\begin{tabular}{cc}
\hline Perspectiva $_{\mathbf{j}}$ & Peso $\left(W p_{j}\right)$ \\
\hline Finanzas & 0,24 \\
Stakeholders & 0,29 \\
Procesos internos & 0,26 \\
Crecimiento y aprendizaje & 0,21 \\
\hline
\end{tabular}

Fuente: Elaboración propia.

igual forma, por lo que su importancia obedece a las dependencias entre las perspectivas.

Se conformaron cuatro matrices, una para cada perspectiva del $B S C$ y su dependencia con las tres restantes. Se emitieron un total de doce criterios, tres por cada matriz, con una inconsistencia que osciló entre 0 y 0,07 .

El resultado de las ponderaciones de las ocho matrices anteriores calculadas en el SuperDecisions arrojó los pesos relativos de las perspectivas (Tabla 6).

Como se puede apreciar la perspectiva más importante para las PyMCE es la de stakeholders, que incluye 
el cumplimiento de la legislación, satisfacción de todas las stakeholders en el desempeño ambiental.

Una vez ponderada las perspectivas, se procede a emitir juicios sobre la importancia de los indicadores de cada perspectiva. Para ello se formaron 4 matrices, una por perspectiva. Las perspectivas de "Finanzas" y "Stakeholders" con cuatro indicadores cada una y doce juicios emitidos y en los casos de "Stakeholders" y "Proceso internos" con diez por cada una.

Igualmente basados en las relaciones causales identificadas por el grupo de expertos entre los indicadores definidos en las perspectivas que se muestran en la Figura 4, se establecieron las comparaciones a pares de cada indicador con los de su perspectiva (relación interna) y las relaciones externas, de los indicadores con indicadores de otra perspectiva. En este paso se pone de manifiesto la principal ventaja del $A N P$, que permite captar las relaciones entre las alternativas (indicadores) formando modelos más complejos y reales.

Con los pesos locales de los indicadores dentro de cada perspectivas y tomando en consideración las relaciones externas identificadas; se calcularon los pesos globales de cada indicador en la perspectiva correspondiente (Tabla 7).
Calculado los pesos de las perspectivas y los indicadores, se homogenizaron y normalizaron los indicadores ambientales definidos y medidos por el grupo de trabajo. Para la homogenización y normalización se utilizó la Fórmula 3.

Esta fórmula ramificada permite tratar simultáneamente indicadores de maximizar y minimizar llevándolos a una unidad común. Los sobre-cumplimiento de las metas se acotan al valor máximo posible en ambos tipos de indicadores.

$$
\boldsymbol{R}_{i j}=\left\{\begin{array}{c}
\frac{x_{i j}}{\text { meta }\left\{x_{i j}\right\}} \text { sielindicador satisface "amayorvalormejor" } \\
1 \text { si } x_{i j} \geq \text { meta }\left\{x_{i j}\right\} \text { "amayorvalormejor" } \\
\frac{\text { meta }\left\{x_{i j}\right\}}{\left\{x_{i j}\right\}} \text { siel indicador satisface "amenorvalormejor" } \\
1 \text { si } x_{i j} \leq \text { meta }\left\{x_{i j}\right\} \text { "amenorvalormejor" }
\end{array}\right.
$$

$\mathrm{R}_{\mathrm{ij}}$ : Valor normalizado del indicador i de la perspectiva $\mathrm{j}$. xij: valor del indicador a normalizar: valor entre 0 y 1 . $\mathrm{i}=$ número de la perspectiva: de 1 a 4 .

$\mathrm{j}=$ número del indicador: de $1 \mathrm{a} \mathrm{n}$.

En la Tabla 8 se muestran los valores $R_{i j}$ de los indicadores, calculados sobre las metas definidas por

Tabla 7. Pesos de los indicadores por perspectivas.

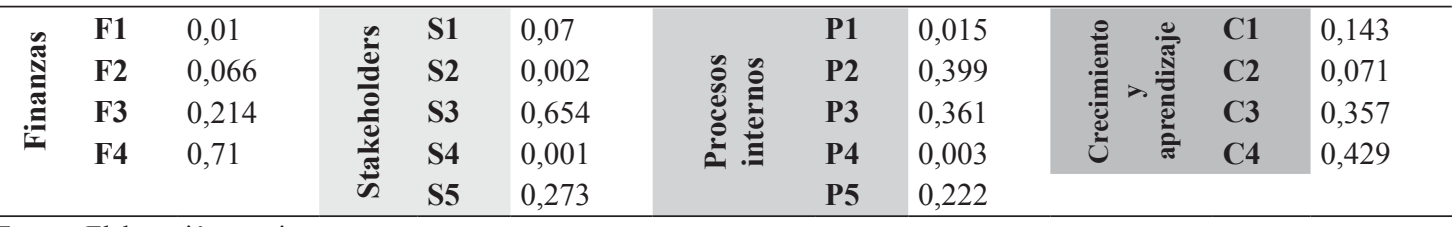

Fuente: Elaboración propia.

Tabla 8. Valores $\mathrm{R}_{\mathrm{ij}}$ de los indicadores de las centrales homogenizados y normalizados por perspectivas.

\begin{tabular}{|c|c|c|c|c|c|}
\hline & & CEF1 & CEF2 & CED1 & CED2 \\
\hline \multirow{4}{*}{ IP $_{\mathbf{F}}$} & F1 & 0,8 & 0,99 & 0,88 & 1 \\
\hline & F2 & 0,8 & 1 & 1 & 1 \\
\hline & F3 & 0,8 & 0,88 & 0,9 & 0,93 \\
\hline & F4 & 0,71 & 1 & 1 & 1 \\
\hline \multirow{5}{*}{$\mathbf{I P}_{\mathrm{S}}$} & S1 & 0,71 & 1 & 1 & 1 \\
\hline & S2 & 0,43 & 1 & 0,71 & 1 \\
\hline & S3 & 0,895 & 0,917 & 0,896 & 0,917 \\
\hline & S4 & 1 & 0,71 & 1 & 0,71 \\
\hline & S5 & 0,75 & 0,88 & 0,85 & 0,94 \\
\hline \multirow{5}{*}{$\mathbf{I P}_{\mathbf{P}}$} & P1 & 0,99 & 1 & 0,99 & 0,8 \\
\hline & P2 & 0,82 & 0,86 & 0,76 & 0,9 \\
\hline & P3 & 0,9 & 0,85 & 0,82 & 0,84 \\
\hline & P4 & 0,76 & 0,81 & 0,77 & 0,88 \\
\hline & P5 & 0,99 & 0,99 & 0,98 & 0,99 \\
\hline \multirow{4}{*}{$\mathbf{I P}_{\mathrm{C}}$} & C1 & 0,815 & 0,8 & 1 & 1 \\
\hline & $\mathrm{C2}$ & 0,43 & 0,71 & 0,43 & 0,43 \\
\hline & C3 & 0,83 & 0,93 & 0,84 & 0,91 \\
\hline & $\mathrm{C4}$ & 0,85 & 0,93 & 0,9 & 1 \\
\hline
\end{tabular}

Fuente: Elaboración propia. 
la dirección y los valores de los indicadores usando la Formula 3, el grupo de trabajo y los expertos en las cuatro PyMCE.

En la Figura 5 se muestran los resultados de los cálculos de los índices de las cuatro perspectivas del $B S C$ por central eléctrica.

Haciendo uso de la Fórmula 1 se calcularon los índices globales de desempeño ambiental para cada PyMCE.

\subsection{Fase 5: Análisis de los resultados}

Como se puede apreciar en la Figura 6, existe una diferencia significativa del orden de diez puntos porcentuales, en cuanto al IGDA de las CEF1 y CEF2. Los subíndices con menores valores numéricos en CEF1 son el IP $\mathrm{F}_{\mathrm{F}}$ y el IP $\mathrm{C}_{\text {. }}$

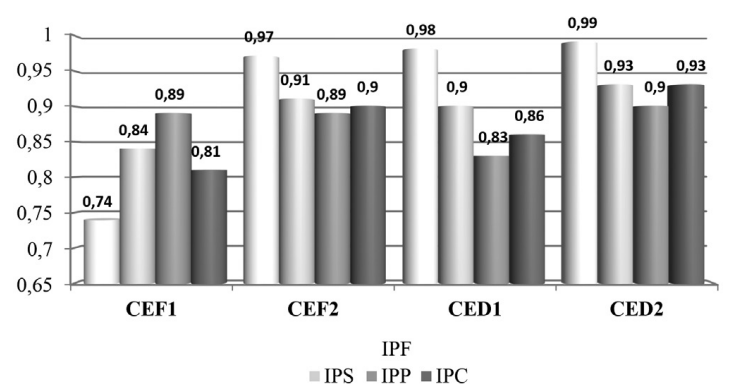

Figura 5. Índices por perspectiva de cada CE. Fuente: Elaboración propia.

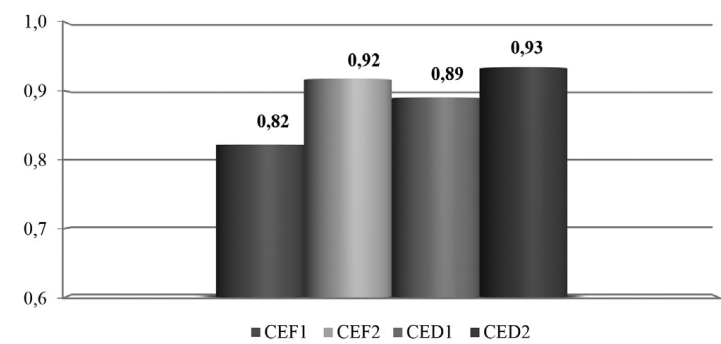

Figura 6. $I G D A$ de las cuatro PyMCE. Fuente: Elaboración propia.
En lo relacionado a las CED1 y CED2 se puede apreciar una diferencia de cuatro puntos porcentuales. Los subíndices con menor valor de estas PyMCE son $\mathrm{IP}_{\mathrm{P}}$ e IP $\mathrm{C}_{\mathrm{P}}$ los dos pertenecientes a CED1.

Para evaluar el desempeño ambiental a través del IGDA se utilizó la escala definida en la Tabla 9.

Para la confección de la escala se trabajó con cuatro expertos de gestión ambiental pertenecientes a la unidad de Supervisión y Control de la Ciencia y el Medio Ambiente del CITMA (Ministerio de Ciencia Tecnología y Medio Ambiente) y tres profesores universitarios. La escala está basada en la eficiencia del cumplimiento de las metas ambientales de la organización. Inicialmente se fijó, bajo consenso, un valor a partir del cual puede ser considerado malo el cumplimiento global de las metas $(0,65)$, se tomó como base varios escenarios posibles del índice. Para los rangos desde 0,65 a 1y apoyados en las nueve unidades en que se divide la escala de dominación de Saaty quedaron definidos los niveles de evaluación. Vale la pena destacar que la escala diseñada tiene como objetivo facilitar a los decisores haciendo uso de valores numéricos, la visualización de avances y retrocesos del cumplimiento de las metas organizacionales.

Utilizando la escala de evaluación de desempeño definida, se evaluaron las PyMCE quedando de la siguiente forma: CEF1 regular, CEF2, CED1 y CED fueron evaluadas de bien aunque en todos los casos existen posibilidades de mejora del desempeño ambiental.

De la descomposición del IGDA se pueden evidenciar los puntos débiles que más afectan el desempeño de las PyMCE y reorientar los esfuerzos de la organización hacia los indicadores en peores condiciones. Para esto se introduce el análisis de los potenciales de mejora de los indicadores (Fórmula 4) que conforman el IGDA.

$$
\text { Potencial demejora }_{\mathbf{i j}}=W p_{j} * W i_{i j} *\left(1-R_{i j}\right) * 100
$$

$W p_{i}$ : El peso relativo de la perspectiva $\mathrm{j}$.

Tabla 9. Escala de evaluación del desempeño ambiental.

\begin{tabular}{ccl}
\hline Saaty & Rango & \multicolumn{1}{c}{ Nivel de evaluación } \\
\hline $8-9$ & $0,95 \leq \boldsymbol{I} \boldsymbol{G} \boldsymbol{D} \boldsymbol{A} \leq 1$ & $\begin{array}{l}\text { Muv bien: el desempeño ambiental se ajusta muy bien a las metas } \\
\text { ambientales definidas por la entidad. }\end{array}$ \\
\hline $6-7$ & $0,85 \leq \boldsymbol{I} \boldsymbol{G D} \boldsymbol{A}<0,95$ & $\begin{array}{l}\text { Bien: el desempeño ambiental se ajusta bien a las metas ambientales } \\
\text { definidas con algunas posibilidades de mejora. }\end{array}$ \\
\hline $4-5$ & $0,75 \leq \boldsymbol{I} \boldsymbol{G D} \boldsymbol{A}<0,85$ & $\begin{array}{l}\text { Regular: el desempeño ambiental se ajusta regular a las metas ambientales } \\
\text { y tiene posibilidades de mejoras significativas. }\end{array}$ \\
\hline $2-3$ & $0,65 \leq \boldsymbol{I G D} \boldsymbol{A}<0,75$ & $\begin{array}{l}\text { Deficiente: el desempeño ambiental es deficiente con respecto a las metas } \\
\text { ambientales definidas y tiene muchas oportunidades de mejoras. }\end{array}$ \\
\hline 1 & $0,65<\boldsymbol{I G D \boldsymbol { A }}$ & $\begin{array}{l}\text { Mal: el desempeño ambiental es malo respecto a las metas ambientales } \\
\text { definidas y tiene grandes oportunidades de mejoras. }\end{array}$ \\
\hline
\end{tabular}

Fuente: Elaboración propia. 

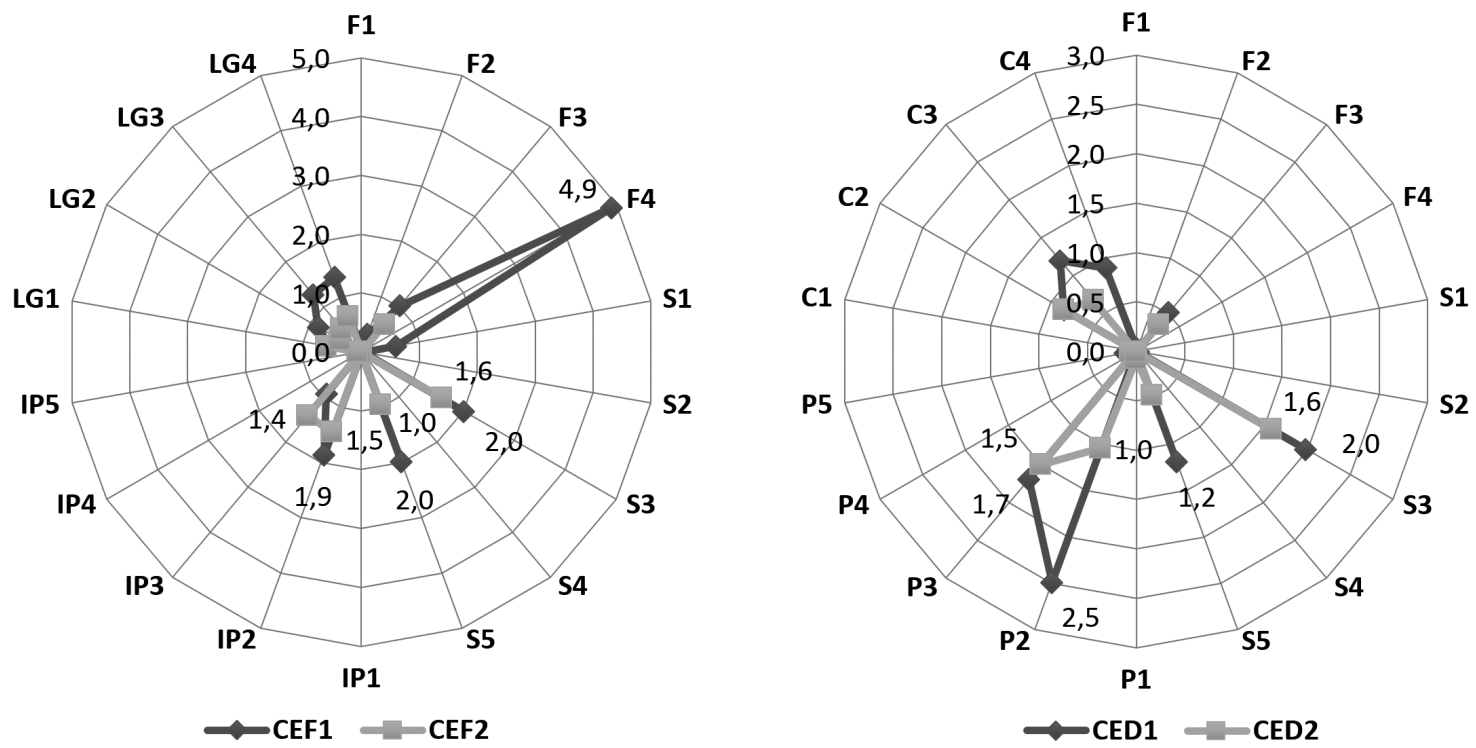

Figura 7. Potenciales de mejora de los indicadores ambientales. Fuente: Elaboración propia.

$W i_{i j}$ : El peso relativo del indicador i en la perspectiva j. $R_{i j}$ : Valor normalizado del indicador i de la perspectiva j. Potencial de mejora $_{i j}$ : Máxima contribución porcentual posible del indicador i de la perspectiva j al IGDA.

En la Figura 7 se pueden observar los indicadores de mayor potencial de mejora en cada PyMCE.

En la CEF1 los puntos débiles detectados fueron las multas (F4), cumplimiento de las regulaciones (S3) y lodos y aguas residuales (P2). En la CEF2 los principales problemas están referidos a S3, P2 y consumo de agua (P3).

En lo relacionado a la CED1 los indicadores de cumplimiento de las regulaciones, consumo de agua $\mathrm{y}$ aguas residuales los de mayor potencial de mejora y en CED2 los indicadores con mayor potencial son: aguas residuales, cumplimiento de las regulaciones, consumo de agua por $\mathrm{kW}$ y trabajos de mejoras ambientales generados por trabajadores $(\mathrm{C} 2)$.

\section{Conclusiones y trabajos futuros}

En el artículo se propone un procedimiento que permite evaluar el desempeño ambiental de una organización, estableciendo una línea de acción para seleccionar, recopilar, analizar, integrar y evaluar los indicadores ambientales empresariales, que respondan a las políticas, estrategias y aspectos ambientales más relevantes de la organización. El procedimiento quedó estructurado en cinco fases: análisis estratégico ambiental, inventario de los procesos, selección de indicadores ambientales, cálculo del índice global de desempeño ambiental y revisión y mejora.

Un aporte de la investigación fue el índice global de desempeño ambiental, que integra en un valor único los indicadores de desempeño, de gestión y el cumplimiento de la legislación, en los subíndices de las perspectivas de un BSC: "Finanzas", "Stakeholders", "Procesos internos" y "Crecimiento y aprendizaje". Además se hace uso del método multi-criterio como $A H P$ y $A N P$ para establecer las prioridades de las perspectivas e indicadores que componen el IGDA. La combinación de todas estas técnicas y métodos pueden ayudar en la naturaleza compleja de los fenómenos ambientales que hacen necesario la integración de los indicadores en un índice agregado donde se tengan en cuenta las posibles relaciones que puedan existir entre ellos.

La aplicación del procedimiento se llevó a cabo en cuatro pequeñas y medianas centrales eléctricas cubanas de la GD, lo que permitió demostrar la factibilidad de aplicación. Se identificaron los principales puntos críticos y oportunidades de mejoras en el desempeño ambiental; facilitando la retroalimentación en relación al cumplimiento de las estrategias ambientales, políticas y metas de la organización.

La principal fortaleza de la propuesta de este artículo radica en la estructuración coherente del procedimiento que orienta a la organización sobre qué debe medir, basado en las estrategias ambientales; combinando los juicios de los expertos con la medición de los indicadores de desempeño, de gestión y de legalidad en el $I G D A$, el que puede ser evaluado de modo continuo, visualizando avances y retrocesos en el cumplimiento de las metas ambientales y del desempeño ambiental. Otro elemento importante es la inclusión de las percepciones de las stakeholders en la evaluación a través del uso del BSC que permite traducir las estrategias hasta los niveles operativos de la jerarquía organizacional. 
Como continuidad de la investigación, se están analizando nuevos objetos de estudio para la aplicación del procedimiento tanto en el propio sector energético como en otros sectores productivos, permitiendo establecer un benchmarking interno. Igualmente se está considerando la posibilidad de desarrollar una herramienta informática que facilite: el almacenamiento de la información, el cálculo del $I G D A$ y la generación de reportes del comportamiento de los diferentes indicadores asociados al desempeño ambiental de la organización.

\section{Agradecimientos}

Los autores quieren agradecer al Prof. Dr.-Ing. habil. Jorge Marx-Gómez por su ayuda a lo largo del proceso de revisiones. También agradecer el apoyo de la Red de Investigación Interdisciplinaria sobre Desarrollo Sostenible (DEVSUS) y el proyecto Eureka SD (DEVSUS número de acuerdo 2013-2591), que es soportado por el programa de Erasmus Mundus de la Unión Europea.

\section{Referencias}

Bieker, T., \& Waxenberger, B. (2001). Sustainability Balanced Scorecard and Business Ethicas. Developing a Balanced Scorecard for Integrity Management. In 10th Conference of the Greening of Industry Network (pp. 1-24). Göteborg. Recuperado el 22 de abril de 2011, de http://www.alexandria.unisg.ch/export/DL/17767.pdf.

Blanc, I., Friot, D., Margni, M., \& Jolliet, O. (2008). Towards a new index for environmental sustainability based on a DALY weighting approach. Sustainable Development, 16(4), 251-260. http://dx.doi.org/10.1002/sd.376.

Broche-Fernández, Y., \& Ramos-Gómez, R. (2010). Procedimiento para la gestión de residuos generados en instalaciones hoteleras cubanas. Retos Turísticos, 9(3), 13-19.

Campos, L., \& Selig, P. M. (2002). SGADA - Sistema de Gestão e Avaliação do Desempenho Ambiental: a aplicação de um modelo de SGA que utiliza o balanced scorecard (BSC). Reading (Sunderland), 8(6), 1-23. [Edición Especial].

Chiang, C. M., \& Lai, C. M. (2002). A study on the comprehensive indicator of indoor environment assessment for occupants' health in Taiwan. Building and Environment, 37(4), 387-392. http://dx.doi.org/10.1016/ S0360-1323(01)00034-8.

Comoglio, C., \& Botta, S. (2012). The use of indicators and the role of environmental management systems for environmental performances improvement: a survey on ISO 14001 certified companies in the automotive sector. Journal of Cleaner Production, 20(1), 92-102. http:// dx.doi.org/10.1016/j.jclepro.2011.08.022.

Conesa-Fernández, V. (1995). Guía metodológica para la evaluación de impacto ambiental (3 ed.). Madrid: Ediciones Mundi-Prensa.
Creswell, J. W. (2007). Projeto de pesquisa: métodos qualitativo, quantitativo e misto (2 ed.). Porto Alegre: Artmed.

Damjan, K., \& Glavic, P. (2005). How to compare companies on relevant dimensions of sustainability. Ecological Economics, (55), 551-563.

Dias-Sardinha, I., \& Reijnders, L. (2005). Evaluating environmental and social performance of large Portuguese companies: a balanced scorecard approach. Business Strategy and the Environment, 14(2), 73-91. http:// dx.doi.org/10.1002/bse.421.

Diewert, W. E. (1976). Exact and superlative index numbers. Journal of Econometrics, 4(2), 115-145. http://dx.doi. org/10.1016/0304-4076(76)90009-9.

Epstein, M., \& Wisner, P. (2001). Using a balanced scorecard to implement sustainability. Environmental Quality Management, 11(2), 1-10. http://dx.doi.org/10.1002/ tqem. 1300.

Figge, F., Hahn, T., Schaltegger, S., \& Wagner, M. (2002). The sustainability balanced scorecard -linking sustainability management to business strategy. Business Strategy and the Environment, 11(5), 269-284. http://dx.doi. org/10.1002/bse.339.

García-Fernández, J. M. (2002). Algunas reflexiones sobre el desarrollo de la gestión ambiental cubana.Cub@: Medio ambiente y Desarrollo, 2(2), 1-8.

Global Reporting Initiative - GRI. (2006). Sustainability reporting guidelines (Version 3.0). Amsterdam: GRI. Recuperado el 19 de noviembre de 2010, de https:// www.globalreporting.org/resourcelibrary/G3-GuidelinesIncl-Technical-Protocol.pdf.

Gunningham, N. (2009). Shaping corporate environmental performance: a review. Environmental Policy and Governance, 19(4), 215-231. http://dx.doi.org/10.1002/ eet. 510 .

Henri, J.-F., \& Journeault, M. (2008). Environmental performance indicators: an empirical study of Canadian manufacturing firms. Journal of Environmental Management, 87(1), 165-176. http://dx.doi.org/10.1016/j. jenvman.2007.01.009. PMid:17368921.

Hermans, E., Van den Bossche, F., \& Wets, G. (2008). Combining road safety information in a performance index. Accident; Analysis and Prevention, 40(4), 13371344. http://dx.doi.org/10.1016/j.aap.2008.02.004. PMid:18606264.

Hernández, C. (2010). Modelo de gerenciamento da logística reversa integrado às questões estratégicas das organizações (Tesis doctoral). Universidade Estadual Paulista, Faculdade de Engenharia de Guaratinguetá, Guaratinguetá.

Hernández, C., Marins, F. A. S., Rocha, P. D., \& Duran, J. A. R. (2010). Using AHP and ANP to Evaluate the relation between reverse logistics and corporate performance in Brazilian Industry. Brazilian Journal of Operations \& Production Management, 7(2), 47-62. 
Herva, M., Franco, A., Carrasco, E. F., \& Roca, E. (2011). Review of corporate environmental indicators. Journal of Cleaner Production, 19(15), 1687-1699. http://dx.doi. org/10.1016/j.jclepro.2011.05.019.

Hsu, C.-W., Hu, A. H., Chiou, C.-Y., \& Chen, T.-C. (2011). Using the FDM and ANP to construct a sustainability balanced scorecard for the semiconductor industry. Expert Systems with Applications, 38(10), 12891-12899. http://dx.doi.org/10.1016/j.eswa.2011.04.082.

Hubbard, G. (2009). Measuring organizational performance: beyond the triple bottom line. Business Strategy and the Environment, 18(3), 177-191. http://dx.doi.org/10.1002/ bse. 564 .

Ilinitch, A. Y., Soderstrom, N. S. \& Thomas, T. E. (1998). Measuring corporate environmental performance. Journal of Accounting and Public Policy, 17(4-5), 383-408.

International Organization for Standardization - ISO. (2005). NC-ISO 14031. Gestión ambiental, evaluación del desempeño ambiental: directrices. La Habana: Oficina Nacional de Normalización.

Isaac-Godínez, C. L., Díaz Aguirre, S., La Rosa Baez, M., Hernández Díaz, R., Hevia Lanier, F., \& Gómez Báez, J. (2010). Indicadores para la evaluación del desempeño ambiental de los Centros de Educación Superior. Revista del Centro Nacional de Investigaciones Cientificas, 41, 1-12.

Jasch, C. (2000). Environmental performance evaluation and indicators. Journal of Cleaner Production, 8(1), 79-88.

Lakatos, E. M., \& Marconi, M. A. (1986). Metodología científica. São Paulo: Atlas.

Lee, W.-S., Huang, A. Y. H., Chang, Y.-Y., \& Cheng, C.-M. (2011). Analysis of decision making factors for equity investment by DEMATEL and Analytic Network Process. Expert Systems with Applications, 38(7), 8375-8383. http://dx.doi.org/10.1016/j.eswa.2011.01.027.

Lober, D. (1996). Evaluating the environmental performance of corporations. Journal of Managerial Issues, 8(2), 184-205.

Luz, S. O. C., Sellitto, M. A., \& Gomes, L. P. (2006). Medição de desempenho ambiental baseada em método multicriterial de apoio à decisão: estudo de caso na indústria automotiva. Gestão \& Produção, 13(3), 557-570. http://dx.doi.org/10.1590/S0104-530X2006000300016.

Medel, F., García, L., Enriquez, S., \& Anido, M. (2011). Reporting models for corporate sustainability in SMEs. In P. Golinska, M. Fertsch \& J. Marx-Gómez (Eds.), Information technologies in environmental engineering (pp. 407-418, Environmental Science and Engineering, 3). Heidelberg: Springer Berlin Heidelberg. Recuperado el 13 de marzo de 2011, de http://link.springer.com/ch apter/10.1007\%2F978-3-642-19536-5_32.

Möller, A., \& Schaltegger, S. (2005). The sustainability balanced scorecard as a framework for eco-efficiency analysis. Journal of Industrial Ecology, 9(4), 73-83. http://dx.doi.org/10.1162/108819805775247927.
Neely, A., Gregory, M., \& Platts, K. (1995). Performance measurement system design: a literature review and research agenda. International Journal of Operations \& Production Management, 4(15), 80-116. http://dx.doi. org/10.1108/01443579510083622.

Oliveira, J. H. (2002). MAIS: Método para Avaliação de Indicadores de Sustentabilidade Organizacional (Tesis doctoral). Universidade Federal de Santa Catarina, Florianopolis.

Olsthoorn, X., Tyteca, D., Wehrmeyer, W., \& Wagner, M. (2001). Environmental indicators for business: a review of the literature and standardisation methods. Journal of Cleaner Production, 9(9), 453-463. http://dx.doi. org/10.1016/S0959-6526(01)00005-1.

Ormazabal, F. J., \& Larrañaga, E. (1999). Guía de indicadores medioambientales para la empresa (pp. 30-48). Berlin: Ministerio Federal de Medio Ambiente de Alemania.

Paoli, D., \& Moraes, L. A. D. F. (2011). Apoio multicritério à decisão como subsídio à gestão ambiental: o caso da Aggreko Brasil. Gestão \& Produção, 18(2), 379-390. http://dx.doi.org/10.1590/S0104-530X2011000200012.

Parra, V. L. R. (2005). Gestión de la información como herramienta para la evaluación del desempeño ambiental. Cub@: Medio Ambiente y Desarrollo, 5(9), 1-12.

Pearson, J., \& Barnes, T. (1999). Improve environmental performance through community action. EcoManagement and Auditing, 6(2), 76-79. http://dx.doi. org/10.1002/(SICI)1099-0925(199906)6:2<76::AIDEMA97>3.0.CO;2-U.

Puolamaa, M., Kaplas, M., \& Reinikainen, T. (1996). Index of environmental friendliness: a methodological study. Helsinki: Statistics Finland.

Ramos, T., \& Melo, J. (2006). Developing and implementing an environmental performance index for the portuguese military. Business Strategy and the Environment, 15(2), 71-86. http://dx.doi.org/10.1002/bse.440.

Rao, P., Singh, A. K., O’Castillo, O., Intal, P. S., Jr., \& Sajid, A. (2009). A metric for corporate environmental indicators... for small and medium enterprises in the Philippines. Business Strategy and the Environment, 18(1), 14-31. http://dx.doi.org/10.1002/bse.555.

Raupp, F. (2012). Modelo de autoavaliação do desempenho ambiental para a agroindústria: o caso da indústria sucroalcooleira do Mato Grosso do Sul (Tesis doctoral). Universidade Federal de Santa Catarina, Florianópolis.

Saaty, R. W., \& Saaty, T. L. (2003). The Analytic Hierarchy Process (AHP) for decision making and the Analytic Network Process (ANP) for decision making with dependence and feedback. Pittsburgh: SuperDecisions. Recuperado el 13 de marzo de 2011, de http://www. superdecisions.com/demos_tutorials.php3.

Saaty, T. L. (1980). The analytic hierarchy process planning, priority setting, resource allocation. New York: McGraw-Hill.

Saaty, T. L. (1996). Decision making with dependence and 
feedback: the analytic network process. Pittsburgh: RWS Publications.

Saaty, T. L. (2003). The seven pillars of the analytic hierarchy process. In T. L. Saaty \& L. G. Vargas (Eds.) Multiple criteria decision making in the new millennium (Cap. 2, pp. 23-40, Lecture Notes in Economics and Mathematical Systems, 507). Heidelberg: SpringerVerlag Berlin Heidelberg.

Sagastume-Gutiérrez, A., Van Caneghem, J., Cogollos Martínez, J. B., \& Vandecasteele, C. (2012). Evaluation of the environmental performance of lime production in Cuba. Journal of Cleaner Production, 31, 126-136. http://dx.doi.org/10.1016/j.jclepro.2012.02.035.

Sellitto, M., Borchardt, M., \& Medeiros-Pereira, G. (2010). Modelagem para avaliação de desempenho ambiental em operações de manufatura. Gestão \& Produção, 17(1), 95-109.

Singh, K., Murty, H. R., Gupta, S. K., \& Dikshit, A. K. (2007). Development of composite sustainability performance index for steel industry. Ecological
Indicators, 7(3), 565-588. http://dx.doi.org/10.1016/j. ecolind.2006.06.004.

Tangen, S. (2005). Analysing the requirements of performance measurement systems. Measuring Business Excellence, 9(4), 46-54. http://dx.doi.org/10.1108/13683040510634835.

Wang, J.-Y., Wang, B., \& Zhao, Q.-C. (2006). Application of Balanced Scorecard and Analytic Network Process to Enterprise Performance Evaluation. Industrial Engineering Journal, 1, 4-17.

Yüksel, I., \& Dagdeviren, M. (2010). Using the fuzzy analytic network process (ANP) for Balanced Scorecard (BSC): A case study for a manufacturing firm. Expert Systems with Applications, 37(2), 1270-1278. http:// dx.doi.org/10.1016/j.eswa.2009.06.002.

Zhou, P., Ang, B. W., \& Poh, K. L. (2006). Comparing aggregating methods for constructing the composite environmental index: An objective measure. Ecological Economics, 59(3), 305-311. http://dx.doi.org/10.1016/j. ecolecon.2005.10.018. 